\title{
HIFI $\alpha$ is an independent prognostic factor for overall survival in advanced primary epithelial ovarian cancer - a study of the OVCAD Consortium
}

\author{
This article was published in the following Dove Press journal: \\ OncoTargets and Therapy \\ II September 2014 \\ Number of times this article has been viewed
}

\author{
Elena loana Braicu' \\ Hrvoje Luketina' \\ Rolf Richter' \\ Dan Cacsire Castillo-Tong ${ }^{2}$ \\ Sandrina Lambrechts ${ }^{4}$ \\ Sven Mahner ${ }^{5}$ \\ Nicole Concin ${ }^{6}$ \\ Monika Mentze' \\ Robert Zeillinger ${ }^{2,3}$ \\ Ignace Vergote ${ }^{4}$ \\ Jalid Sehouli' \\ 'Department of Gynecology, European \\ Competence Center for Ovarian \\ Cancer, Charité - Universitätsmedizin \\ Berlin, Berlin, Germany; ' $D e p a r t m e n t$ \\ of Obstetrics and Gynecology, \\ Comprehensive Cancer Center, \\ Medical University of Vienna, ${ }^{3}$ Ludwig \\ Boltzmann Cluster Translational \\ Oncology, General Hospital of \\ Vienna, Vienna, Austria; ${ }^{4}$ Department \\ of Obstetrics and Gynecology, \\ Universitaire Ziekenhuizen Leuven, \\ Katholieke Universiteit Leuven, Leuven, \\ Belgium; ${ }^{5}$ Department of Gynecology \\ and Gynecologic Oncology, University \\ Medical Center Hamburg-Eppendorf, \\ Hamburg, Germany; ${ }^{6}$ Department of \\ Obstetrics and Gynecology, Innsbruck \\ Medical University, Innsbruck, Austria
}

Correspondence: Elena loana Braicu Department of Gynecology, European Competence Center for Ovarian Cancer, Campus Virchow Clinic, Charité - University Hospital of Berlin, Augustenburger Platz I, I 3353 Berlin, Germany

Email elena.braicu@charite.de
Purpose: Hypoxia is a common phenomenon encountered in solid cancers, leading to chemotherapy resistance and therefore to aggressiveness of the disease. The homeostatic response to hypoxia is mediated by hypoxia-inducible factor-1 (HIF-1). The aim of this study was to investigate the impact of HIF $1 \alpha$ in patients with primary epithelial ovarian cancer.

Methods: In this multicentric study, 275 patients with advanced primary epithelial ovarian cancer were included. All patients underwent cytoreductive surgery with maximal surgical effort and adjuvant platinum-based chemotherapy. HIF $1 \alpha$ expression was analyzed in tissue lysates, using an enzyme-linked immunosorbent assay.

Results: HIF $1 \alpha$ was detected in $79.3 \%$ of the tissue samples. Patients with increased HIF $1 \alpha$ expression (cutoff: $80 \mathrm{pg} / \mathrm{mg}$ protein) in tumoral tissue lysates were more likely to have less favorable survival. HIF $1 \alpha(P=0.009$, hazard ratio [HR] 2.505, 95\% confidence interval [95\% CI] 1.252-5.013) together with International Federation of Gynecology and Obstetrics (III versus IV) $(P=0.013$, HR $0.540,95 \%$ CI $0.332-0.878)$, histology $(P=0.007$, HR 2.748, 95\% CI 1.315-5.743), presence of peritoneal carcinomatosis ( $P=0.014$, HR 2.176, 95\% CI 1.170-4.046), residual tumor mass ( $P=0.017$, HR 1.641, 95\% CI 1.091-2.468), and response to platinum-based chemotherapy $(P<0.001$, HR $8.131,95 \%$ CI 5.13-12.88) were independent prognosis factors for overall survival. The independent prognostic factors for progression-free survival were International Federation of Gynecology and Obstetrics stage $(P=0.01)$, histological subtypes $(P=0.016)$, and presence of peritoneal carcinomatosis $(P<0.05)$.

Conclusion: HIF $1 \alpha$ overexpression in ovarian cancer is associated with poor overall survival, underlining the importance of hypoxia in this angiogenesis driven disease.

Keywords: HIF1 $\alpha$, surgical outcome, platinum response, survival, primary epithelial ovarian cancer, predictive factors

\section{Introduction}

Ovarian cancer is the leading cause of death from gynecological malignancies, with more than half of patients being diagnosed in the advanced stage. The overall 5-year survival rate is around 40\% in International Federation of Gynecology and Obstetrics (FIGO) stages III/IV and 90\% in FIGO stage I. ${ }^{1}$

The current standard of treatment for primary ovarian cancer is the combination of optimal cytoreductive surgery and platinum-based chemotherapy. Despite state of the art treatment, more than $50 \%$ of the patients will relapse and consequently die from this disease. ${ }^{2}$ A curative approach is only possible in the primary situation. The aim of targeted 
therapy is to prolong the tumor-free status achieved after the primary treatment. Recent data showed that some antiangiogenic therapies, eg, bevacizumab, are able to prolong progression-free survival, thus no improvement in overall survival has been shown yet. ${ }^{3,4}$ There is an urgent need to identify new targets, and also biomarkers able to predict platinum response.

No macroscopic residual tumor mass after cytoreductive surgery is one of the major prognostic and predictive factors. Despite radical surgery, maximal tumor reduction cannot always be achieved. A neoadjuvant approach could have a benefit in this subgroup of patients, although data are controversial. ${ }^{5-8}$ Nevertheless, there are no effective biomarkers to predict surgical outcome in primary ovarian cancer patients.

HIF $1 \alpha$ has been reported to be an important predictor of tumor progression for several types of solid cancers. ${ }^{9-13}$ HIF $1 \alpha$ is a major regulator of cell adaptation to hypoxic stress and plays a critical role in oncogenesis and angiogenesis. ${ }^{14}$ This protein regulates the transcription of a number of genes involved in diverse biological functions such as proliferation, migration, invasion, apoptosis, and angiogenesis.

Immunohistochemical analysis of human cancer biopsies has shown that HIF $1 \alpha$ is overexpressed in several solid malignancies, including breast, colon, lung, gastric, skin, and renal carcinomas, compared with their respective normal tissues. ${ }^{15,16}$

Like other tumors, overexpression of HIF $1 \alpha$ has been detected in epithelial ovarian cancer. ${ }^{15}$ Data regarding the clinical impact of HIF $1 \alpha$ in ovarian cancer is still limited. Although HIF $1 \alpha$ is detected in all epithelial ovarian cancer histological subtypes, higher HIF $1 \alpha$ expression in clear-cell carcinomas has been reported. ${ }^{17,18}$

The role of HIF $1 \alpha$ in the development of new targeted therapies has been suggested by recent studies. ${ }^{19,20}$ Nakai et al stated that HIF $1 \alpha$-expressing tumors had a significantly higher response rate to postoperative platinum-based chemotherapy than tumors without HIF1 $\alpha$ expression. ${ }^{21}$

However, the prognostic significance of HIF $1 \alpha$ overexpression in ovarian cancer is still controversial. ${ }^{21-24}$ Until now, only limited data exist regarding the HIF $1 \alpha$ predictive value in epithelial ovarian cancer.

The aim of this study was to investigate the impact of HIF1 $\alpha$ expression in predicting surgical outcome, platinum response, and survival in patients with advanced primary epithelial ovarian cancer.

\section{Patients and methods}

This analysis was performed within the Sixth Framework Program European project "Ovarian Cancer - Diagnosis of a Silent Killer" (OVCAD). At five comprehensive centers for ovarian cancer treatment, 275 patients with advanced primary epithelial ovarian cancer (FIGO stage II-IV) were enrolled.

All patients gave their written informed consent before tissue samples were collected. Patients were recruited from 2005-2008 in the Department of Gynecology at Charité - Medical University Berlin (Berlin, Germany), Department of Gynecologic Oncology, University Hospital Leuven (Leuven, Belgium), Department of Obstetrics and Gynecology, Medical University of Vienna (Vienna, Austria), Department of Gynecology, University Medical Center Hamburg-Eppendorf (Hamburg, Germany), and Department of Gynecology and Obstetrics, Innsbruck Medical University (Innsbruck, Austria). This study was approved by each local ethics committee (EK207/2003, ML2524, HEK190504, EK366, and EK260).

Only patients with histologically confirmed ovarian cancer were included in the study. Due to better prognosis and sometimes different therapy strategies, FIGO stage I patients were excluded. All patients underwent cytoreductive surgery with maximal surgical effort and platinum-based chemotherapy. Inclusion and exclusion criteria for the study were reported in a recent publication. ${ }^{25}$ An online database was used for the documentation of clinical, histopathological, and follow-up data. Residual tumor load was defined as negative if macroscopically absent. Overall survival was defined as the time from diagnosis of ovarian cancer to tumor-related death or last contact. Progression-free survival was defined as the interval from diagnosis to progression of disease or death, whereby progression was defined as an increase in the nadir serum CA-125 level of at least two samples according to the Gynecological Cancer Intergroup criteria or radiological diagnosis according to the Response Evaluation Criteria in Solid Tumors (RECIST) criteria.

\section{Documentation of clinical data}

The tumor spread within the abdominal cavity was documented prospectively using the Intraoperative Mapping of Ovarian cancer - "IMO" tool. ${ }^{26}$ The documentation of residual tumor mass was also assessed prospectively at the end of the surgery with an interview with the main surgeon. All data were documented in a validated online database.

The FIGO 2006 classification was used for clinical staging, and the Gynecologic Oncology Group criteria were used for histologic grading. ${ }^{27}$ Response to treatment and diagnosis of recurrence was determined according to RECIST criteria 
or according to CA-125 variations (Gynecological Cancer Intergroup criteria) during follow-up. ${ }^{27,28}$

Response to first-line chemotherapy was defined according to the last time patients received platinum-based compounds. Patients developing relapse within the first 6 months after platinum based chemotherapy were classified as platinum-resistant; patients developing relapse after 6 months or more were classified as responders. ${ }^{29}$

\section{Collection of tumor tissue}

Tumor tissue was removed from the patients during surgery and prior to chemotherapy treatment. Samples were snap frozen and stored in liquid nitrogen. At a later time, the tissue was processed to obtain lysate for protein measurement.

About $30 \mathrm{mg}$ tumor tissue was homogenized with a Mikro-Dismembrator and lysed in $1 \mathrm{~mL}$ Nucleic Acid Purification Lysis Solution (Thermo Fisher Scientific, Waltham, MA, USA).

\section{HIFI $\alpha$ enzyme-linked immunosorbent assay (ELISA)}

HIF $1 \alpha$ expression in tissue lysates was measured using the HIF $1 \alpha$ ELISA kit (R\&D Systems, Inc., Minneapolis, MN, USA) according to the manufacturer's protocol. The assay was performed in duplicate for the calibrators, controls, and patient samples. The appropriate controls were within the ranges provided by the manufacturer.

\section{Statistical analysis}

The clinical data were collected and entered in an online database. The statistical analysis was performed using IBM ${ }^{\mathbb{R}}$ SPSS $^{\circledR}$ Statistics 21.0 (IBM Corporation, Armonk, NY, USA) at Charité - Medical University Berlin.

The prognostic factors used in the survival analysis were as follows: age at first diagnosis, FIGO stage, histological subtype, histological grade, presence and volume of ascites, residual tumor mass after surgery, peritoneal dissemination, and responses to platinum-based chemotherapy.

Kruskal-Wallis test, Spearman's rank correlation, Kendall's tau, and Mann-Whitney $U$ test were used to assess the associations between HIF $1 \alpha$ and other variables.

Receiver operating characteristic curve analysis was performed to evaluate the predictive accuracy of HIF $1 \alpha$ expression for discriminating patients with maximal versus suboptimal residual tumor mass after surgery and platinum responders versus nonresponders.

Median survival and $95 \%$ confidence intervals ( $95 \% \mathrm{CI}$ ) were estimated according to the Kaplan-Meier method.
The log-rank tests were used for univariate statistical comparisons and the Cox proportional hazard model was used to evaluate significant predictors of survival. Adjusted hazard ratios (HR) and $95 \% \mathrm{CI}$ for prognostic factors were calculated. A two-tailed $P$-value $<0.05$ was considered statistically significant.

\section{Results}

\section{Baseline characteristics}

Most of the patients (86.2\%) were diagnosed with serous ovarian cancer. Optimal debulking, ie, no evidence of macroscopic tumor residuals, was reported in more than $60 \%$ of the patients. Patients' characteristics are presented in Table 1, and are reported in greater detail elsewhere..$^{25}$

Table I Patient's clinical and pathologic characteristics

\begin{tabular}{|c|c|}
\hline Parameter & Primary ovary cancer \\
\hline $\begin{array}{l}\text { Ovarian cancer samples included to the } \\
\text { multicenter study, } \mathrm{n}(\%)\end{array}$ & $275(100.0)$ \\
\hline $\begin{array}{l}\text { Age at time of diagnosis, median (range), } \\
\text { years }\end{array}$ & $58(18-85)$ \\
\hline \multicolumn{2}{|l|}{ Histological type, n (\%) } \\
\hline Serous & $237(86.2)$ \\
\hline Endometrioid & $13(4.7)$ \\
\hline Mixed/others* & $25(9.1)$ \\
\hline \multicolumn{2}{|l|}{ Tumor stage, n (\%) } \\
\hline FIGO II & $15(5.4)$ \\
\hline FIGO III & $212(77.1)$ \\
\hline FIGO IV & $48(17.5)$ \\
\hline \multicolumn{2}{|l|}{ Lymph node status, $\mathrm{n}(\%)$} \\
\hline No & $65(23.6)$ \\
\hline $\mathrm{NI}$ & $143(52)$ \\
\hline $\mathrm{Nx}$ & $67(24.4)$ \\
\hline \multicolumn{2}{|l|}{ Distant metastatic spread, n (\%) } \\
\hline Mo & $142(5 \mid .6)$ \\
\hline MI & $49(17.8)$ \\
\hline Mx & $84(30.5)$ \\
\hline \multicolumn{2}{|l|}{ Grading, n (\%) } \\
\hline Well differentiated & $10(3.6)$ \\
\hline Moderately differentiated & $64(23.3)$ \\
\hline Poorly differentiated & $200(72.7)$ \\
\hline Unknown & $\mathrm{I}(0.4)$ \\
\hline CA-I 25 level, n (\%) & $258(93.8)$ \\
\hline Preoperatively, median (range), U/mL & $1,500.24(7-37,820)$ \\
\hline \multicolumn{2}{|l|}{ Ascites volume, $\mathrm{n}(\%)$} \\
\hline None & $66(24)$ \\
\hline$\leq 500 \mathrm{~mL}$ & $110(40)$ \\
\hline$>500 \mathrm{~mL}$ & $100(36)$ \\
\hline \multicolumn{2}{|l|}{ Peritoneal carcinomatosis, n (\%) } \\
\hline Yes & $186(67.6)$ \\
\hline No & $89(32.4)$ \\
\hline \multicolumn{2}{|l|}{ Postoperative residual tumor mass, n (\%) } \\
\hline Macroscopic tumor free & $188(68.4)$ \\
\hline$\leq \mathrm{I} \mathrm{cm}$ & $48(17.5)$ \\
\hline$>1 \mathrm{~cm}$ & $38(13.8)$ \\
\hline Missing & I (0.4) \\
\hline
\end{tabular}

Note: *Eleven mixed, nine undifferentiated, and two clear-cell carcinomas. Abbreviation: FIGO, International Federation of Gynecology and Obstetrics. 


\section{Expression of $\mathrm{HIFI} \alpha$}

Expression of HIF1 $\alpha$ was confirmed in 218 (79.3\%) patients. No expression of HIF $1 \alpha$ was observed in 57 tissue samples (20.7\%). The median HIF1 $\alpha$ expression was 56.8 (range $0-1,492.31) \mathrm{pg} / \mathrm{mg}$ protein.

\section{Correlation with classical prognostic factors}

No statistically significant association between HIF $1 \alpha$ expression and clinical prognostic factors such as age $(P=0.238)$, volume of ascites $(P=0.866)$, FIGO stage ( $P=0.0896)$, and grading $(P=0.152)$ was found.

Levels of HIF $1 \alpha$ were higher in patients presenting peritoneal carcinomatosis and mucinous and mixed histology, although this correlation retained no statistical significance ( $P=0.065$ and $P=0.071$, respectively).

There was no correlation between HIF $1 \alpha$ and residual tumor mass after cytoreductive surgery $(P=0.342$, area under the curve $0.555,95 \%$ CI $0.471-0.639$ ) or response to platinum-based chemotherapy $(P=1$, area under the curve 0.499, 95\% CI 0.413-0.586) (Table 2).

\section{Survival analysis}

The median follow-up time for patients was 37 months, ranging from 1-69 months; 194 patients developed

Table 2 Correlation with clinical prognostic factors

\begin{tabular}{|c|c|c|}
\hline Clinical parameters & $\begin{array}{l}\text { HIF I } \alpha \text { median } \\
\text { (pg/mg protein) }\end{array}$ & $P$-value \\
\hline \multicolumn{3}{|l|}{ FIGO stage } \\
\hline FIGO stage II & 36.9 & 0.896 \\
\hline FIGO stage III & 35.7 & \\
\hline FIGO stage IV & 34.6 & \\
\hline \multicolumn{3}{|l|}{ Histology } \\
\hline Serous & 34.8 & 0.071 \\
\hline Endometrioid & 33.4 & \\
\hline Mixed & 77.1 & \\
\hline Mucinous & 19.9 & \\
\hline \multicolumn{3}{|l|}{ Grading } \\
\hline GI & 53.4 & 0.152 \\
\hline G2-G3 & 35.1 & \\
\hline \multicolumn{3}{|l|}{ Platinum response } \\
\hline Responder & 35.1 & $\mathrm{I}$ \\
\hline Nonresponder & 35.7 & \\
\hline \multicolumn{3}{|l|}{ Peritoneal carcinomatosis } \\
\hline Present & 38 & 0.065 \\
\hline Absent & 30 & \\
\hline \multicolumn{3}{|l|}{ Ascites } \\
\hline Absent & 34.5 & 0.914 \\
\hline$\leq 500 \mathrm{~mL}$ & 35.1 & \\
\hline$>500 \mathrm{~mL}$ & 37.1 & \\
\hline \multicolumn{3}{|l|}{ Residual tumor mass } \\
\hline Absent & 33.3 & 0.342 \\
\hline Present & 41.5 & \\
\hline
\end{tabular}

Abbreviation: FIGO, International Federation of Gynecology and Obstetrics. recurrent disease and 134 patients died from ovarian cancer.

In order to analyze the role of HIF $1 \alpha$ for survival, the lower and upper quintiles were used as cutoff values. Using the cutoff values of $15 \mathrm{pg} / \mathrm{mg}$ protein and $80 \mathrm{pg} / \mathrm{mg}$ protein, it could be shown that patients presenting HIF $1 \alpha$ tissue concentrations higher than $80 \mathrm{pg} / \mathrm{mg}$ protein would have a shorter overall survival, although this association was not significant within the univariate analysis. When multivariate analysis was performed, HIF $1 \alpha$ concentrations over $80 \mathrm{pg} / \mathrm{mg}$ protein remained an independent prognostic biomarker for overall survival ( $P=0.009$, HR $2.505,95 \%$ CI $1.252-5.013)$ (Figure 1) together with FIGO (III versus IV) $(P=0.013$, HR $0.540,95 \%$ CI $0.332-0.878$ ), histology (mixed versus serous versus endometrioid) $(P=0.007$, HR $2.748,95 \%$ CI 1.315-5.743), presence of peritoneal carcinomatosis ( $P=0.014$, HR $2.176,95 \%$ CI 1.170-4.046), residual tumor mass (present versus absent macroscopically) $(P=0.017$, HR 1.641, 95\% CI 1.091-2.468), and response to platinum-based chemotherapy ( $P<0.001$, HR $8.131,95 \%$ CI $5.13-12.88)$.

FIGO stage ( $P=0.001$, HR $0.478,95 \%$ CI $0.314-0.726)$, presence of peritoneal carcinomatosis $(P<0.001, \mathrm{HR} 2.620$, 95\% CI 1.704-4.027), and histology ( $P=0.021$, HR 2.306, 95\% CI 1.137-4.677) were the only independent predictive factors for progression-free survival.

\section{Discussion}

This study analyzed the role of HIF $1 \alpha$ in primary ovarian cancer. The results showed that patients having tumors that overexpress HIF $1 \alpha$ transcription factor (cutoff $80 \mathrm{pg} / \mathrm{mg}$ protein) are presenting a poorer prognosis.

One of the major bottlenecks in ovarian cancer management is the development of platinum resistance with

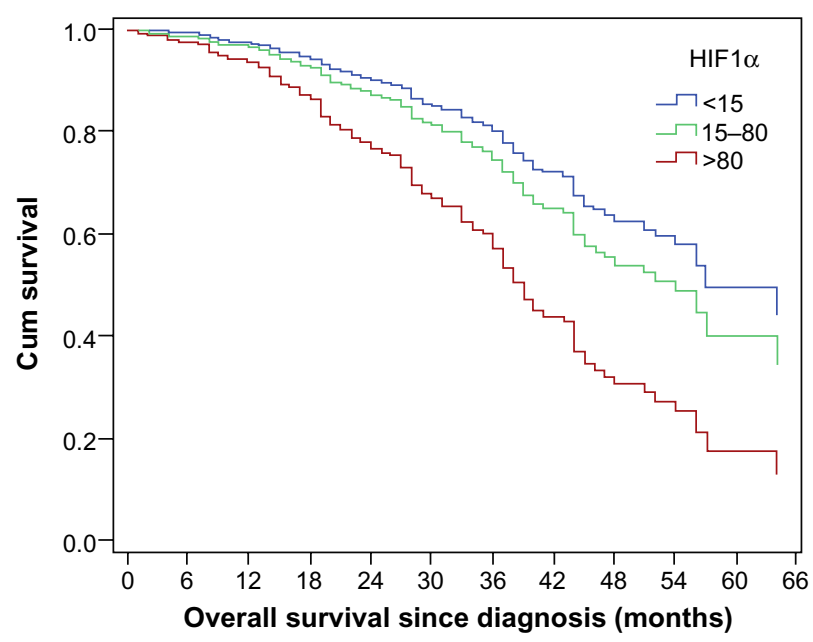

Figure I HIFI $\alpha$ expression and overall survival $(P=0.009$, hazard ratio $2.505,95 \%$ confidence interval 1.252-5.013). 
subsequently increased mortality. The development of new targeted, especially antiangiogenic, drugs has translated into prolonged progression-free survival without evidence of a positive impact on overall survival. The inhibition of neoangiogenesis in ovarian cancer leads to a certain hypoxia of the tumoral cells, and therefore increases apoptosis of tumoral cells. There are no in sufficient data to show a benefit in overall survival for patients being treated with antiangiogenic drugs. This might be caused by the capability of tumor cells to resist even hypoxic conditions.

One of the mechanisms of chemotherapy resistance in solid tumors is the ability to develop a microenvironment caused by a poor and inefficient vascular supply. ${ }^{30}$ The presence of hypoxia has been shown to be a marker for poor prognosis in many cancer types. The resistance to conventional chemotherapeutical drugs is caused by hypoxia-induced reduction in cell proliferation and also by the fact that hypoxic tumor cells reside some distance from the blood vessels, and therefore the drugs won't be able to reach their target. ${ }^{31,32}$ The homeostatic response to hypoxia is mediated by the transcription factor HIF $1 \alpha$, which is unstable in well-oxygenated tissue but becomes stable under hypoxic conditions. ${ }^{33}$ Due to hypoxic conditions in tumoral tissue, as described previously, HIF $1 \alpha$ is an important mediator for tumor invasion, metastasis, and chemotherapy resistance. ${ }^{34}$

This study showed that HIF $1 \alpha$ expression was independent of FIGO stage, age, and grading. Mucinous and mixed epithelial carcinomas showed increased levels of HIF $1 \alpha$, although this association didn't reach statistical significance. Previously published data showed that clear-cell carcinomas exhibit higher HIF $1 \alpha$ levels than serous, mucinous, or endometrioid tumors. ${ }^{35,36}$ Other studies suggest that HIF $1 \alpha$ might be overexpressed in serous ovarian cancer. ${ }^{24}$ The difference in the current results could be caused by low numbers of well-differentiated (3.6\%) and non-serous ovarian cancer patients included within the OVCAD study. More than that, differences in ethnicity might cause differences in the molecular biology of ovarian cancer. It is well known that clear-cell ovarian cancer is more often encountered in Asian women compared to Caucasian women, and the clinical course of the disease is different. Clinical studies showed that chemotherapy or targeted therapy drugs are associated with different response rates and adverse effects in Caucasian and Asian populations. ${ }^{37}$

Results similar to the current study were published by Nakayama et al, who reported that HIF $1 \alpha$ levels were independent of clinical stage, age and histological subtypes. ${ }^{38} \mathrm{HIF} 1 \alpha$ expression was reported to be significantly stronger in good differentiated ovarian cancer. ${ }^{17}$ The current results showed no significant correlation between HIF $1 \alpha$ tissue expression and histological grading, which might be explained by the low number of well-differentiated (G1) ovarian cancers included in this study. There was also no significant influence of HIF $1 \alpha$ expression on response to platinum-based chemotherapy, this being in accordance with some existing results. ${ }^{17}$ However, Nakai et al reported that tumors expressing higher HIF $1 \alpha$ more likely respond to platinum-based chemotherapy compared with tumors with no or poor HIF $1 \alpha$ expression. ${ }^{21}$ The current study analyzed the expression of HIF $1 \alpha$ in 275 patients with FIGO stage II-IV, but Nakai et al's study only included 52 stage III-IV epithelial ovarian cancer patients.

HIF $1 \alpha$ increased tissue values were associated with shorter progression-free survival, but this was not statistically significant, which agrees with recent studies. ${ }^{22,23}$ Daponte et al observed that the median progression-free survival of HIF $1 \alpha$-positive patients was shorter, but not statistically significant. ${ }^{22}$ In lung and colorectal carcinomas, HIF $1 \alpha$ had no impact on patient survival, but overexpression of HIF $2 \alpha$ was a prognostic indicator. ${ }^{39,40}$ In ovarian carcinomas, Birner et al showed that HIF1 $\alpha$ overexpression alone was not a prognostic indicator and became a strong prognostic marker in combination with functional p53 protein. ${ }^{17}$

\section{Conclusion}

The current study showed that HIF $1 \alpha$ tissue expression might impact the overall survival in primary ovarian cancer patients treated by cytoreductive surgery and platinum-based chemotherapy. No correlation between HIF $1 \alpha$ tissue expression and platinum response and no significant statistical difference between HIF $1 \alpha$ expression in different histological subtypes was found. Further studies analyzing the role of this transcriptional factor in a larger prospective cohort of patients are warranted. Studies analyzing the HIF $1 \alpha$ distribution within tumor tissue using immunochemistry are needed.

Drugs interfering with the HIF $1 \alpha$ pathway, such as ganetespib, ${ }^{41}$ are currently under evaluation in clinical studies. Targeting HIF $1 \alpha$ might be a way to improve survival in ovarian cancer patients.

\section{Acknowledgments}

Elena Ioana Braicu is a participant in the Charité Clinical Scientist Program funded by Charite - Universitätsmedizin Berlin and the Berlin Institute of Health.

The OVCAD project was funded by the European commission as FP6 Specific Targeted Research and Innovation Project (contract no 018698). 


\section{Disclosure}

The authors report no conflicts of interest in this work.

\section{References}

1. Ferlay J, Parkin DM, Steliarova-Foucher E. Estimates of cancer incidence and mortality in Europe in 2008. Eur J Cancer. 2010;46(4): 765-781.

2. Gilks CB, Prat J. Ovarian carcinoma pathology and genetics: recent advances. Hum Pathol. 2009;40(9):1213-1223.

3. du Bois A, Floquet A, Kim JW, et al. Randomized, double-blind, Phase III trial of pazopanib versus placebo in women who have not progressed after first-line chemotherapy for advanced epithelial ovarian, fallopian tube, or primary peritoneal cancer (AEOC): results of an international Intergroup trial (AGO-OVAR16). In: Abstracts of the 2013 ASCO Annual Meeting; May 31-June 4, 2013; Chicago, IL, USA. Abstract 5503.

4. Monk BJ, Dalton H, Farley JH, Chase DM, Benjamin I. Antiangiogenic agents as a maintenance strategy for advanced epithelial ovarian cancer. Crit Rev Oncol Hematol. 2013;86(2):161-175.

5. Vergote I, Tropé CG, Amant F, et al. Neoadjuvant chemotherapy or primary surgery in stage IIIC or IV ovarian cancer. $N$ Engl $J$ Med. 2010;363(10):943-953.

6. du Bois A, Marth C, Pfisterer J, et al. Neoadjuvant chemotherapy cannot be regarded as adequate routine therapy strategy of advanced ovarian cancer. Int J Gynecol Cancer. 2012;22(2):182-185.

7. Kehoe S, Hook J, Nankivell M, et al. Chemotherapy or upfront surgery for newly diagnosed advanced ovarian cancer: results from the MRC CHORUS trial. In: Abstracts of the 2013 ASCO Annual Meeting; May 31June 4, 2013; Chicago, IL, USA. Abstract 5500.

8. Sehouli J, Savvatis K, Braicu EI, Schmidt SC, Lichtenegger W, Fotopoulou C. Primary versus interval debulking surgery in advanced ovarian cancer: results from a systematic single-center analysis. Int $J$ Gynecol Cancer. 2010;20(8):1331-1340.

9. Gruber G, Greiner RH, Hlushchuk R, et al. Hypoxia-inducible factor $1 \alpha$ in high-risk breast cancer: an independent prognostic parameter? Breast Cancer Res. 2004;6(3):R191-R198.

10. Kurokawa T, Miyamoto M, Kato K, et al. Overexpression of hypoxiainducible-factor $1 \alpha(\mathrm{HIF}-1 \alpha)$ in oesophageal squamous cell carcinoma correlates with lymph node metastasis and pathologic stage. $\mathrm{Br} J$ Cancer. 2003;89(6):1042-1047.

11. Lidgren A, Hedberg Y, Grankvist K, Rasmuson T, Vasko J, Ljungberg B. The expression of hypoxia-inducible factor $1 \alpha$ is a favorable independent prognostic factor in renal cell carcinoma. Clin Cancer Res. 2005;11(3):1129-1135.

12. Swinson DE, Jones JL, Cox G, Richardson D, Harris AL, O'Byrne KJ. Hypoxia-inducible factor- $1 \alpha$ in non-small-cell lung cancer: relation to growth factor, protease and apoptosis pathways. Int J Cancer. 2004;111(1):43-50.

13. Fillies T, Werkmeister R, van Diest PJ, Brandt B, Joos U, Buerger H. HIF 1- $\alpha$ overexpression indicates a good prognosis in early stage squamous cell carcinomas of the oral floor. BMC Cancer. 2005;5:84.

14. Lu X, Kang Y. Hypoxia and hypoxia-inducible factors: master regulators of metastasis. Clin Cancer Res. 2010;16(24):5928-5935.

15. Zhong H, De Marzo AM, Laughner E, et al. Overexpression of hypoxia-inducible factor $1 \alpha$ in common human cancers and their metastases. Cancer Res. 1999;59(22):5830-5835.

16. Talks KL, Turley H, Gatter KC, et al. The expression and distribution of the hypoxia-inducible factors HIF- $1 \alpha$ and HIF- $2 \alpha$ in normal human tissues, cancers, and tumor-associated macrophages. Am J Pathol. 2000;157(2):411-421.

17. Birner P, Schindl M, Obermair A, Breitenecker G, Oberhuber G. Expression of hypoxia-inducible factor $1 \alpha$ in epithelial ovarian tumors: its impact on prognosis and on response to chemotherapy. Clin Cancer Res. 2001;7(6):1661-1668.
18. Miyazawa M, Yasuda M, Fujita M, et al. Association of hypoxiainducible factor-1 expression with histology in epithelial ovarian tumors: a quantitative analysis of HIF-1. Arch Gynecol Obstet. 2009;279(6):789-796.

19. Anglesio MS, George J, Kulbe H, et al. IL6-STAT3-HIF signaling and therapeutic response to the angiogenesis inhibitor sunitinib in ovarian clear-cell cancer. Clin Cancer Res. 2011;17(8):2538-2548.

20. Yamaguchi K, Mandai M, Oura T, et al. Identification of an ovarian clear-cell carcinoma gene signature that reflects inherent disease biology and the carcinogenic processes. Oncogene. 2010;29(12): 1741-1752.

21. Nakai H, Watanabe Y, Ueda H, Hoshiai H. Hypoxia inducible factor 1- $\alpha$ expression as a factor predictive of efficacy of taxane/ platinum chemotherapy in advanced primary epithelial ovarian cancer. Cancer Lett. 2007;251(1):164-167.

22. Daponte A, Ioannou M, Mylonis I, et al. Prognostic significance of hypoxia-inducible factor $1 \alpha$ (HIF-1 $\alpha$ ) expression in serous ovarian cancer: an immunohistochemical study. BMC Cancer. 2008;8:335.

23. Seeber LM, Horree N, Vooijs MA, et al. The role of hypoxia inducible factor- $1 \alpha$ in gynecological cancer. Crit Rev Oncol Hematol. 2011;78(3):173-184.

24. Osada R, Horiuchi A, Kikuchi N, et al. Expression of hypoxia-inducible factor $1 \alpha$, hypoxia-inducible factor $2 \alpha$, and von Hippel-Lindau protein in epithelial ovarian neoplasms and allelic loss of von HippelLindau gene: nuclear expression of hypoxia-inducible factor $1 \alpha$ is an independent prognostic factor in ovarian carcinoma. Hum Pathol. 2007;38(9):1310-1320.

25. Chekerov R, Braicu I, Castillo-Tong DC, et al. Outcome and clinical management of 275 patients with advanced ovarian cancer International Federation of Obstetrics and Gynecology II to IV inside the European Ovarian Cancer Translational Research Consortium-OVCAD. Int $J$ Gynecol Cancer. 2013;23(2):268-275.

26. Sehouli J, Könsgen D, Mustea A, et al. ["IMO" - intraoperative mapping of ovarian cancer]. Zentralbl Gynakol. 2003;125(3-4): 129-135. German.

27. Rustin GJ, Vergote I, Eisenhauer E, et al. Definitions for response and progression in ovarian cancer clinical trials incorporating RECIST 1.1 and CA 125 agreed by the Gynecological Cancer Intergroup (GCIG). Int J Gynecol Cancer. 2011;21(2):419-423.

28. Therasse P, Arbuck SG, Eisenhauer EA, et al. New guidelines to evaluate the response to treatment in solid tumors. J Natl Cancer Inst. 2000;92(3):205-216.

29. Friedlander M, Butow P, Stockler M, et al. Symptom control in patients with recurrent ovarian cancer: measuring the benefit of palliative chemotherapy in women with platinum refractory/resistant ovarian cancer. Int J Gynecol Cancer. 2009;19(Suppl 2):S44-S48.

30. Vaupel P, Kelleher DK. Blood flow and oxygenation status of prostate cancers. Adv Exp Med Biol. 2013;765:299-305.

31. Chen Y, Zhang L, Pan Y, Ren X, Hao Q. Over-expression of semaphorin $4 \mathrm{D}$, hypoxia-inducible factor- $1 \alpha$; and vascular endothelial growth factor is related to poor prognosis in ovarian epithelial cancer. Int J Mol Sci. 2012;13(10):13264-13274.

32. Shannon AM, Bouchier-Hayes DJ, Condron CM, Toomey D. Tumour hypoxia, chemotherapeutic resistance, and hypoxia-related therapies. Cancer Treat Rev. 2003;29(4):297-307.

33. Zhang JJ, Wu HS, Wang L, Tian Y, Zhang JH, Wu HL. Expression and significnace of TLR4 and HIF $1 \alpha$ in pancreatic ductal adenocarcinoma. World J Gastroenterol. 2010;16(23): 2881-2888.

34. Shi CY, Fan Y, Liu B, Lou WH. HIF1 contributes to hypoxia-induced pancreatic cancer cells invasion via promoting QSOX1 expression. Cell Physiol Biochem. 2013;32(3):561-568.

35. Cheng JC, Klausen C, Leung PC. Hypoxia-inducible factor $1 \alpha$ mediates epidermal growth factor-induced down-regulation of E-cadherin expression and cell invasion in human ovarian cancer cells. Cancer Lett. 2013;329(2):197-206. 
36. Lee S, Garner EI, Welch WR, Berkowitz RS, Mok SC. Over-expression of hypoxia-inducible factor $1 \alpha$ in ovarian clear-cell carcinoma. Gynecol Oncol. 2007;106(2):311-317.

37. Tam KF, Chan YM, Ng TY, Wong LC, Ngan HY. Ethnicity is a factor to be considered before dose planning in ovarian cancer patients to be treated with topotecan. Int J Gynecol Cancer. 2006;16(1):135-139.

38. Nakayama K, Kanzaki A, Hata K, et al. Hypoxia-inducible factor $1 \alpha$ $($ HIF-1 $\alpha)$ gene expression in human ovarian carcinoma. Cancer Lett. 2002;176(2):215-223.

39. Giatromanolaki A, Koukourakis MI, Sivridis E, et al. Relation of hypoxia inducible factor $1 \alpha$ and $2 \alpha$ in operable non-small-cell lung cancer to angiogenic/molecular profile of tumours and survival. Br J Cancer. 2001;85(6):881-890.
40. Yoshimura H, Dhar DK, Kohno H, et al. Prognostic impact of hypoxiainducible factors $1 \alpha$ and $2 \alpha$ in colorectal cancer patients: correlation with tumor angiogenesis and cyclooxygenase- 2 expression. Clin Cancer Res. 2004;10(24):8554-8560.

41. Xiang L, Gilkes DM, Chaturvedi P, et al. Ganetespib blocks HIF-1 activity and inhibits tumor growth, vascularization, stem cell maintenance, invasion, and metastasis in orthotopic mouse models of triple-negative breast cancer. J Mol Med (Berl). 2014;92(2):151-164.

\section{Publish your work in this journal}

OncoTargets and Therapy is an international, peer-reviewed, open access journal focusing on the pathological basis of all cancers, potential targets for therapy and treatment protocols employed to improve the management of cancer patients. The journal also focuses on the impact of management programs and new therapeutic agents and protocols on

\section{Dovepress}

patient perspectives such as quality of life, adherence and satisfaction. The manuscript management system is completely online and includes a very quick and fair peer-review system, which is all easy to use. Visit http://www.dovepress.com/testimonials.php to read real quotes from published authors.

Submit your manuscript here: http://www.dovepress.com/oncotargets-and-therapy-journal 